\title{
Systemic Inflammation Impairs Mood Function by Disrupting the Resting-State Functional Network in a Rat Animal Model Induced by Lipopolysaccharide Challenge
}

\author{
Xia Zhu $\mathbb{D}^{1,2}$ Mu-Huo Ji, ${ }^{3}$ Shu-Ming $\mathrm{Li}^{3}{ }^{3}$ Bin $\mathrm{Li}^{3}{ }^{3} \mathrm{Li}$ Mei, ${ }^{4}$ and Jian-Jun Yang $\mathbb{D}^{1,5}$ \\ ${ }^{1}$ Department of Anesthesiology, Jinling Clinical Medical College of Nanjing Medical University, Nanjing, Jiangsu, China \\ ${ }^{2}$ Department of Anesthesiology, The Affiliated Lianyungang Oriental Hospital of Kangda College of Nanjing Medical University, \\ Lianyungang, Jiangsu, China \\ ${ }^{3}$ Department of Anesthesiology, Zhongda Hospital, Medical School, Southeast University, Nanjing, Jiangsu, China \\ ${ }^{4}$ Department of Anesthesiology, Guangdong General Hospital, Guangdong Academy of Medical Sciences, Guangzhou, China \\ ${ }^{5}$ Department of Anesthesiology, The First Affiliated Hospital of Zhengzhou University, Zhengzhou, Henan, China
}

Correspondence should be addressed to Jian-Jun Yang; yjyangjj@126.com

Received 27 November 2018; Revised 15 March 2019; Accepted 23 April 2019; Published 9 May 2019

Academic Editor: Daniela Caccamo

Copyright (c) 2019 Xia Zhu et al. This is an open access article distributed under the Creative Commons Attribution License, which permits unrestricted use, distribution, and reproduction in any medium, provided the original work is properly cited.

\begin{abstract}
Background. Systemic inflammation impairs cognitive performance, yet the brain networks mediating this process remain to be elucidated. The purpose of the current study was to use resting-state functional magnetic resonance imaging (fMRI) to explore changes in the functional connectivity in a lipopolysaccharide- (LPS-) induced systemic inflammation animal model. Materials and Methods. We used the regional homogeneity (ReHo) method to examine abnormal brain regions between the control and LPS groups and then considered them as seeds of functional connectivity analysis. Results. Compared with the control group, our study showed that (1) LPS impaired mood function, as reflected by a depression-like behavior in the forced swim test; (2) LPS induced significantly increased ReHo values in the anterior cingulate cortex (ACC) and caudate putamen $(\mathrm{CPu})$; (3) the ACC seed showed increased functional connectivity with the retrosplenial cortex, superior colliculus, and inferior colliculus; and (4) the right $\mathrm{CPu}$ seed showed increased functional connectivity with the left $\mathrm{CPu}$. Linear regression analysis showed a LPS-induced depression-like behavior which was associated with increased ReHo values in the ACC and right CPu. Moreover, the LPS-induced depression-like behavior was related to increased functional connectivity between the right $\mathrm{CPu}$ and left $\mathrm{CPu}$. Conclusion. This is the first study to show that systemic inflammation impairs mood function that is associated with an altered resting-state functional network based on ReHo analysis, providing evidence of the abnormal regional brain spontaneous activity which might be involved in inflammation-related neurobehavioral abnormalities.
\end{abstract}

\section{Introduction}

Sepsis-associated encephalopathy (SAE) is a central nervous system (CNS) complication induced by systemic inflammation in response to bacterial lipopolysaccharide (LPS) or other endotoxic bacterial cell wall components without direct brain infection [1-3]. Of note, SAE can affect up to $70 \%$ of patients with severe sepsis, leading to long-term neurobehavioral abnormalities, poor quality of life, and even increased mortality [2]. However, the pathophysiology of SAE remains largely to be elucidated [4-6]. Given the relevance of a disturbed neural circuit in the pathophysiology of many neuropsychiatric disorders [7-9], understanding the neural basis of inflammation-mediated affective and cognitive impairment is essential for its diagnosis and treatment.

Functional magnetic resonance imaging (fMRI) based on blood oxygen level-dependent (BOLD) contrast is a useful technique that has been extensively applied to study the brain and its functional organization in both healthy and disease states [7-9]. Although resting-state fMRI reflects spontaneous neuronal activity and functional connectivity indirectly, 
it requires no stimulus-response sequence and is highly replicable and thus receives increased attention among researchers investigating cognitively compromised patients [10] and depression [11]. Studies using fMRI have consistently found that neural networks within and between brain structures facilitate some functional purpose or neuronal processing $[7,8,12]$. On the contrary, disrupted or altered resting-state brain activities are frequently observed in Alzheimer's disease [13] and depression [14] and in patients following chemotherapy [15]. Strikingly, previous neuroimaging studies on sickness behavior have demonstrated that systemic inflammation alters the functional connectivity of brain networks within the human brain at rest $[16,17]$. In these studies, one main approach being applied to characterize the resting-state networks is the seed-based functional connectivity in a priori defined subnetworks [16]. However, this method explored the fMRI signal from the aspect of temporal correlation but not regional brain spontaneous activity. On the other hand, regional homogeneity (ReHo) measures the functional coherence of a given voxel with its nearest neighbors [18], which can reflect the temporal homogeneity of neural activity and effectively evaluate resting-state brain activity. Currently, ReHo is a potentially powerful tool for investigating the alterations in resting-state brain activity, thereby complementing the information provided by functional connectivity analysis. Indeed, the ReHo algorithm is proven to be useful in detecting the changes in a variety of diseases such as major depression [19], Parkinson's disease [20], and schizophrenia [21]. As the BOLD signal of fMRI may reflect neural activity, abnormal ReHo is possibly relevant to the changes of temporal aspects of neural activity in the regional brain [18]. However, there is a paucity of information available on brain functional connectivity changes after peripheral LPS challenge based on ReHo analysis.

In light of these findings, we used the ReHo method to examine abnormal brain regions between the control and LPS groups. Regions showing ReHo differences were selected as seeds for further functional connectivity analysis. We hypothesized that LPS-induced mood and cognitive impairment involves altered ReHo values and neural connectivity when compared with the control group.

\section{Materials and Methods}

2.1. Animals and Study Protocol. Approval was issued by the Ethics Committee of Zhongda Hospital (Nanjing, China). All the experimental procedures were implemented under the Guide for the Care and Use of Laboratory Animals from the National Institutes of Health (Bethesda, MD, USA). As subjects, thirty-two male Sprague-Dawley rats weighing 320-380 g were purchased from the Animal Center of Jinling Hospital (Nanjing, China) and cultivated in $12 \mathrm{~h}$ light-dark cycles (lighting at 07:00, a.m.) at $24 \pm 1^{\circ} \mathrm{C}$, with free access to water and chow. One rat in the control group was excluded due to obvious head movement, and then fifteen rats in the control group and sixteen rats in the LPS group were included in the final analysis. The diagram of the study protocol is shown in Figure 1(a).
2.2. Systemic LPS Administration and Dosage. In the present study, the rats were injected with either LPS (Escherichia coli endotoxin 0111: B4, Sigma, Lot \# 064M4125V, Shanghai, China; $1 \mathrm{mg} / \mathrm{kg}$ LPS, a single-dose, intraperitoneal injection) or equal-volume $(0.9 \%)$ normal saline (a single-dose, intraperitoneal injection). To maintain circadian effects, all the injections were completed between 8:00 and 9:00 a.m. The dose of LPS was designed as neurobehavioral-abnormalitycausing, but not lethal [22-24].

2.3. Resting-State fMRI Acquisition. MRI was examined by the 7.0 T Bruker PharmaScan MRI scanner (70/16 PharmaScan, Bruker BioSpin GmbH, Germany). A quadrature volume resonator (inner diameter $72 \mathrm{~mm}$ ) was used for radio frequency transmission, and a 4-element surface coil array was used for signal reception. For anesthesia induction, a mixture of isoflurane (5\% for induction and $0.3 \%-0.5 \%$ for maintenance) and dexmedetomidine sedation was used [11]. A nose mask with a bite bar was used to deliver the isoflurane mixture on the MRI bed and to fix the animal in prone position. During the MRI scan, the rat was prostrated on a custom-made holder to minimize head motion, while the respiration rate was controlled between 60 and 80 breaths/min. An echo-planar imaging (EPI) sequence was performed: matrix size $=64 \times 64$, flip angle $=$ $30^{\circ}$, resolution $=0.5 \mathrm{~mm} \times 0.5 \mathrm{~mm}$, slice thickness $=1.0 \mathrm{~mm}$, slice gap $=0, \quad$ repetition time $(\mathrm{TR})=2 \mathrm{~s}, \quad$ echo time $(\mathrm{TE})=$ $18 \mathrm{~ms}$, and volume $=180$. A coplanar T2-weighted scan was also acquired.

2.4. ReHo Calculation and Seed-Based Functional Connectivity Analysis. Preprocessing and analysis of the fMRI data were performed using SPM8 (Functional Imaging Laboratory, Wellcome Department of Cognitive Neurology, London, $\mathrm{UK})$. The ReHo calculation procedure was applied as previously described [18]. This is accomplished on a voxel-by-voxel basis by calculating Kendall's coefficient of concordance for a given voxel time series with those of its nearest 26 neighbors. In our study, we used the ReHo method to examine altered brain regions and considered them as seeds for further functional connectivity analysis, which included the anterior cingulate cortex (ACC) and caudate putamen $(\mathrm{CPu})$.

First, the images shot at the first 10 time points were eliminated, then the images were realigned in a time series. Those on which the head moved by more than $0.1 \mathrm{~mm}$ and 1 degree were excluded. The left qualified were registered to a template set which was based on a standard rat brain atlas of Paxinos and Watson. And the voxel of fMRI images was spatially normalized to $3 \mathrm{~mm} \times$ $3 \mathrm{~mm} \times 3 \mathrm{~mm}$ and smoothed with an isotropic Gaussian kernel $(\mathrm{FWHM}=4 \mathrm{~mm})$.

ReHo and functional connectivity analysis was operated on a Resting-State fMRI Data Analysis Toolkit V1.8 software (REST, http://www.restfmri.net). Multiple comparison corrections were conducted by family-wise error rate correction with a cluster-defining threshold $(P=0.001$, two-tailed), relevant to a cluster level of $P=0.05$. 


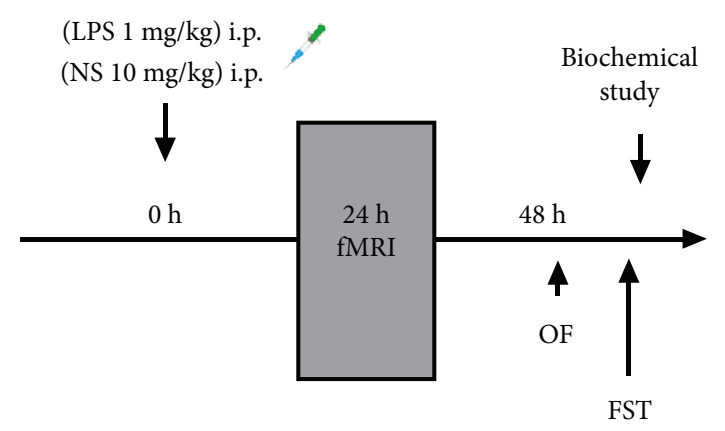

(a)

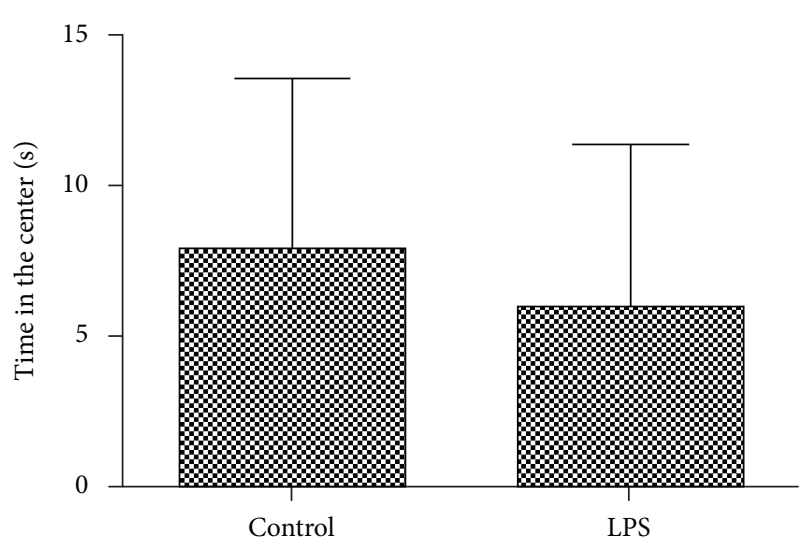

(c)

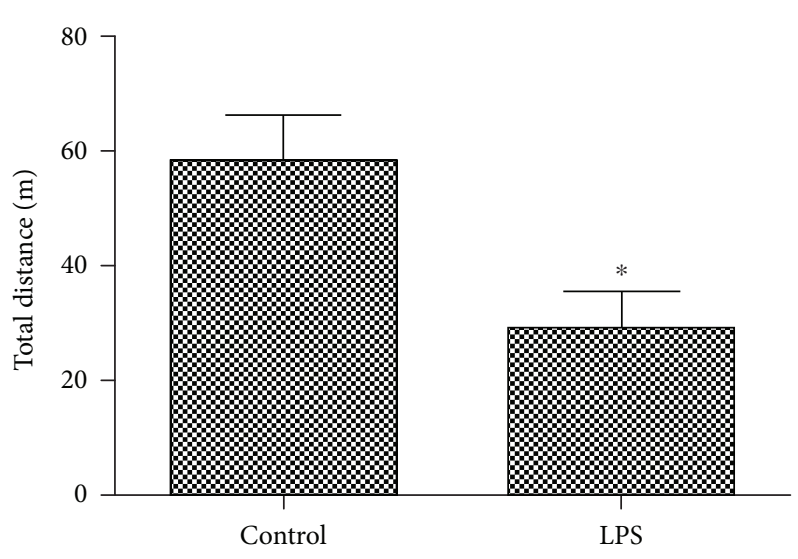

(b)

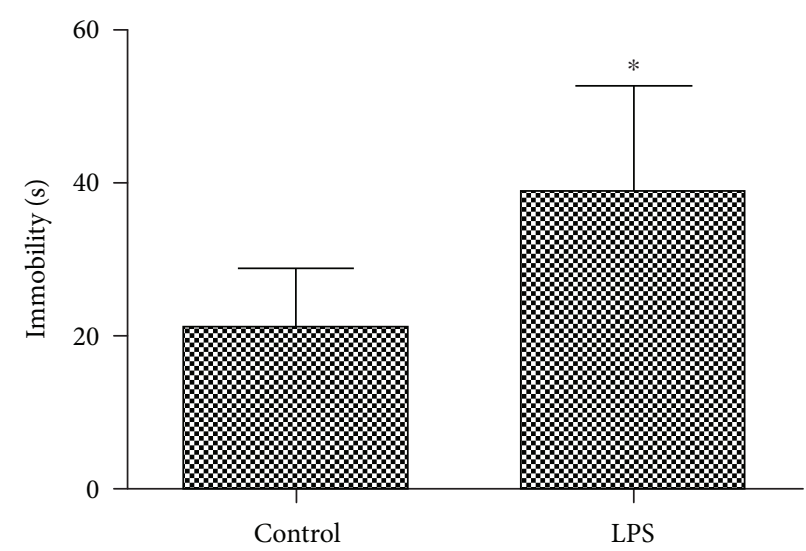

(d)

Figure 1: (a) Experimental design of the study. (b) Effects of LPS on total distance traveled in the open field. (c) Effects of LPS on time spent in the center in the open arena. (d) Effects of LPS on immobility time in the forced swimming test. OF: open field; FST: forced swim test. NS: normal saline; LPS: lipopolysaccharide. ${ }^{*} P<0.05$ vs. control group.

2.5. Neurobehavioral Tests. Behavioral tests were performed as we previously described $[25,26]$.

All devices were provided by Shanghai Softmaze Information Technology Co. Ltd. (Shanghai, China). The rat behavior was videoed by an experienced investigator blind to the test.

2.5.1. Open Field Test. An open field test was performed to evaluate the exploratory behavior and anxiety behavior.

The rats were centered into the black plastic chamber $(100 \mathrm{~cm} \times 100 \mathrm{~cm} \times 40 \mathrm{~cm})$ and allowed to explore it for $5 \mathrm{~min}$. The total distance that a rat migrated and the time it took in the center of the open field were recorded by an experimenter blinded to the test. After one round of test, the arena was deodorized with $75 \%$ alcohol.

2.5.2. Forced Swim Test. Rats were placed singly in a plastic cylinder (30 cm diameter and $80 \mathrm{~cm}$ height) filled with water $\left(23-25^{\circ} \mathrm{C}\right)$ for $6 \mathrm{~min}$, with the immobility scored in the final four minutes only. Time spent immobile (absence of movement except leg kicks to stay afloat) is then used as a measure of behavioral despair and helplessness, a rodent analogue of depressive-like behavior.
2.6. Enzyme-Linked Immunosorbent Assay (ELISA). The rats were deep-anesthetized with $2 \%$ sodium pentobarbital in saline $(60 \mathrm{mg} / \mathrm{kg}$, intraperitoneally; Sigma Chemical Co., St. Louis, MO, USA). The blood was collected transcardially from thoracotomized rats and centrifuged at $4000 \mathrm{rpm}$ for $5 \mathrm{~min}$ at $4^{\circ} \mathrm{C}$. Plasma samples were stored at $-80^{\circ} \mathrm{C}$. The levels of interleukin- $1 \beta$ (IL-1 $\beta$ ), tumor necrosis factor (TNF- $\alpha$ ), IL-6, and brain-derived neurotrophic factor (BDNF) were measured by ELISA kits purchased from Jiancheng Biotechnology (Nanjing, China).

2.7. Statistical Analysis. Data presented as mean \pm standard deviation (S.D.) was analyzed with SPSS 16.0. The Kolmogorov-Smirnov test was used for normally distributed data. Group comparisons were performed by twosample $t$-tests. To determine the relationship between imaging data and depression-like behavior, Pearson's correlation was used. $P<0.05$ was considered statistically significant.

\section{Results}

3.1. LPS Challenge Impaired Mood Function. In the open field test, LPS challenge resulted in a significantly decreased total 


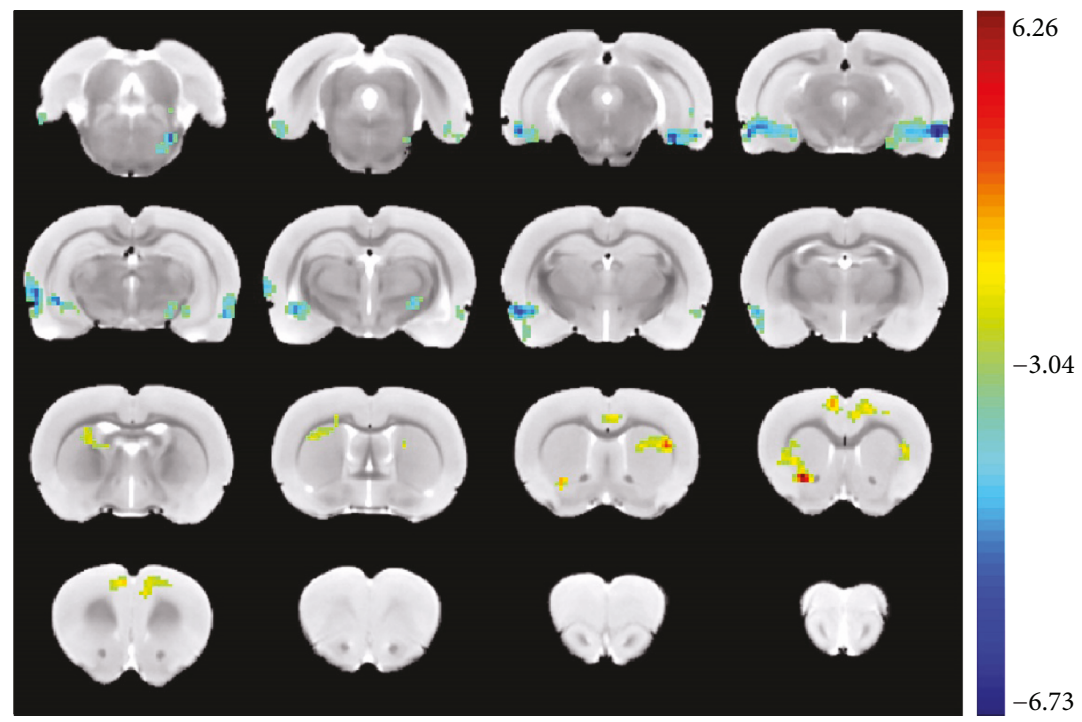

(a)

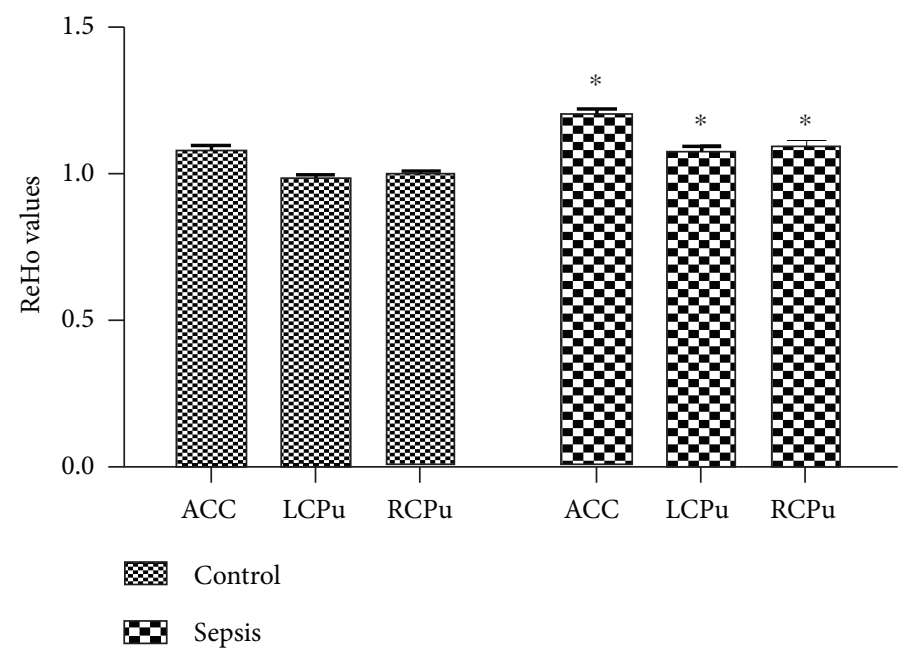

(b)

FIGURE 2: LPS challenge altered ReHo values. (a) The group difference distribution map of the ReHo $(P<0.05$, corrected by family-wise error rate). Significantly increased ReHo are highlighted in the red-yellow heat bar scales; significantly decreased ReHo are highlighted in the blue-green heat bar scales. The ACC seed showed increased functional connectivity with the retrosplenial cortex compared with the control group. (b) The bar graph shows the mean ReHo values of ACC, LCPu, and RCPu with significant between-group differences extracted from the LPS and control groups. ACC: anterior cingulate cortex; LCPu: left caudate putamen; RCPu: right caudate putamen. ${ }^{*} P<0.05$ vs. control group.

distance traveled in the open field arena when compared with the control group $(t=11.048, P<0.001$, Figure $1(\mathrm{~b}))$, suggesting that LPS induced reduced motility and sickness behavior. However, there was no difference in the time spent in the center arena of the open field between the two groups $(t=0.937, P=0.357$, Figure $1(\mathrm{c}))$. In the forced swimming test, immobility of the LPS group was significantly increased when compared with the control group $(t=-4.354, P<$ 0.001 , Figure $1(\mathrm{~d}))$, indicating LPS challenge caused a depression-like behavior.

3.2. LPS Challenge Altered the ReHo Value. Since the main purpose of the study was to evaluate the influence of LPS challenge on cognitive function, we adopted the ReHo method, a data-driven analysis, after which the regions showing ReHo differences between the control and LPS groups were defined as seeds for further functional connectivity analysis. As shown in Figure 2, LPS challenge significantly increased ReHo values in rat brain regions, including the ACC and the $\mathrm{CPu}$ compared with the control group.

3.3. LPS Challenge Altered Functional Connectivity. After the analysis, we selected the ACC and the $\mathrm{CPu}$ as seeds of functional connectivity analysis. Compared with the control group, the ACC seed showed significantly increased 


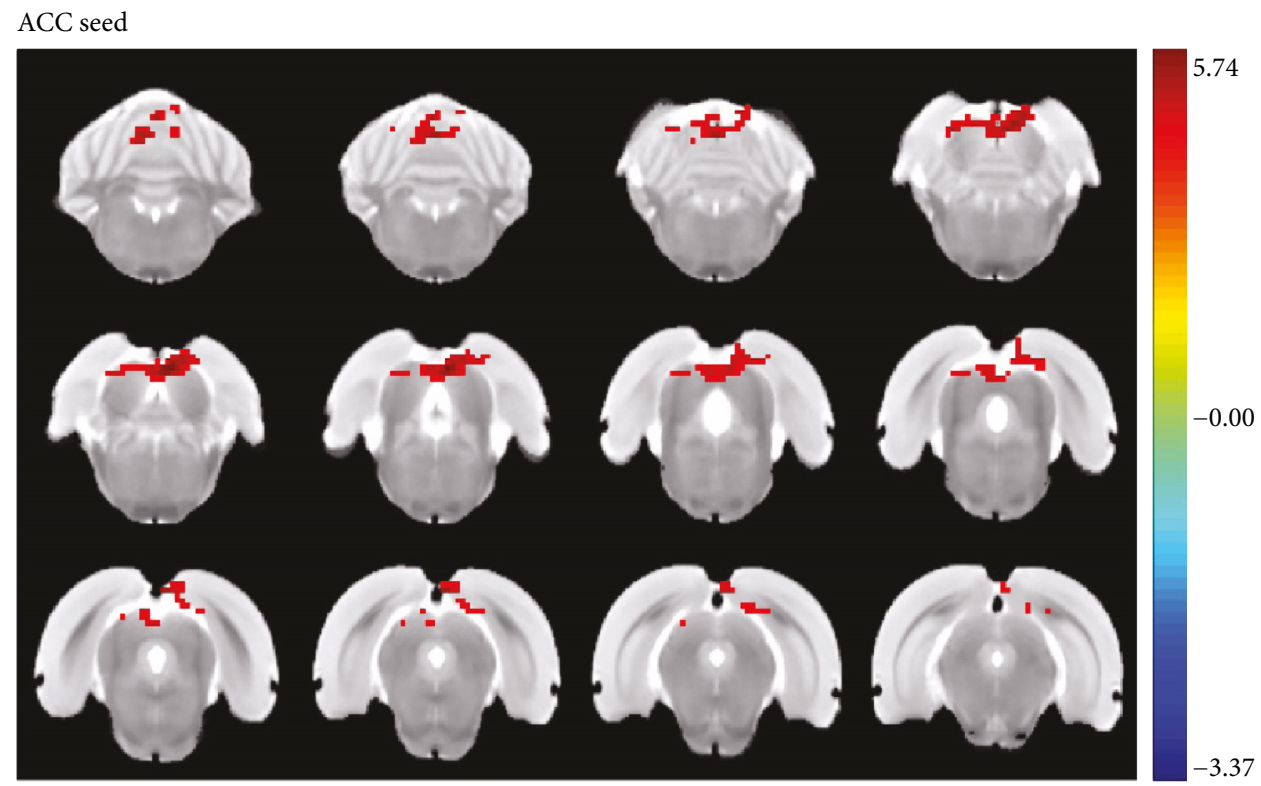

(a)

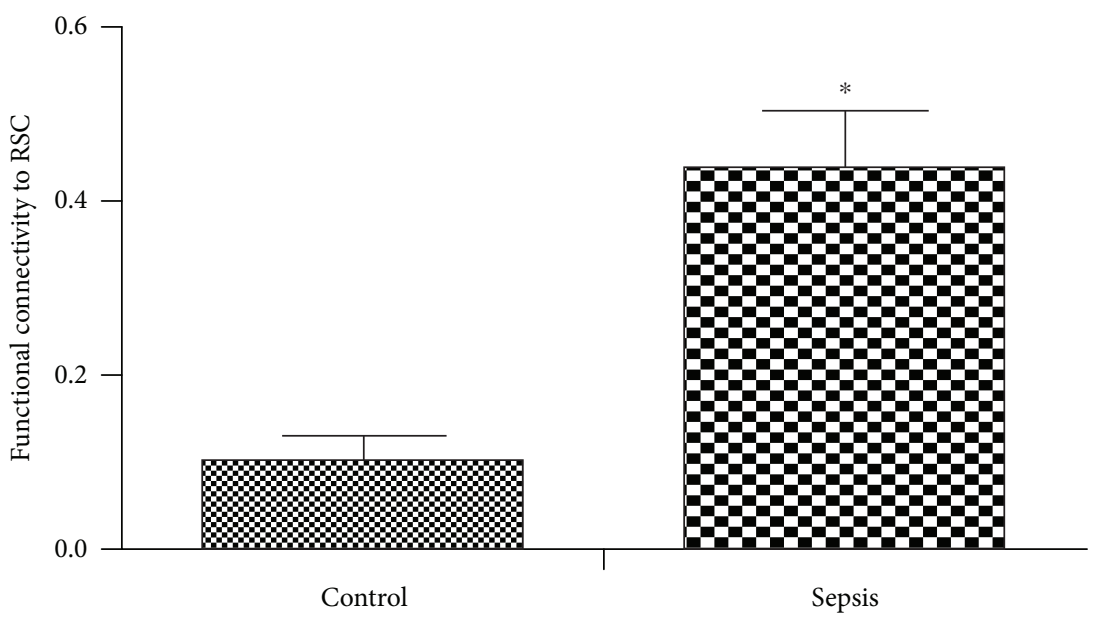

(b)

FIGURE 3: LPS challenge altered functional connectivity between the anterior cingulate cortex and retrosplenial cortex. (a) The group difference distribution map of the FC when the seed was set at the ACC $(P<0.05$, corrected by family-wise error rate). Significantly increased FC are highlighted in the red-yellow heat bar scales. The ACC seed showed increased functional connectivity with the retrosplenial cortex compared with the control group. (b) The bar graph shows the mean FC $Z$ values of RSC with the significant between-group difference extracted from the LPS and control groups. FC: functional connectivity, ACC: anterior cingulate cortex; RSC: retrosplenial cortex. ${ }^{*} P<0.05$ vs. control group.

functional connectivity with the retrosplenial cortex, superior colliculus, and inferior colliculus (Figure 3). In addition, the right $\mathrm{CPu}$ seed showed significantly increased functional connectivity with the left $\mathrm{CPu}$ (Figure 4).

\subsection{LPS Challenge Did Not Change Plasma Proinflammatory} Mediators but Decreased the BDNF Level. As shown in Figures 5(a)-5(c), LPS challenge did not change plasma proinflammatory mediators when performed two days after injection compared with the control group (TNF- $\alpha$ : $t=$ $-0.334, P=0.741$, Figure 5(a); IL-1 $\beta$ : $t=-0.1695, P=$ 0.101 , Figure 5(b); IL-6: $t=-0.673, P=0.506$, Figure 5(c)). However, our study showed that LPS challenge significantly decreased the plasma level of BDNF as compared with the control group $(t=3.783, P=0.001$, Figure $5(d))$.

3.5. Correlation between Depression-Like Behavior and Neuroimaging Data. Within the LPS group, the ReHo values of the ACC and the right $\mathrm{CPu}$ showed significant correlations with the depression-like behavior (Figures 6(a) and 6(b)). Also, the functional connectivity between the right $\mathrm{CPu}$ and the left $\mathrm{CPu}$ was significantly related to depression-like behavior (Figure 6(d)). However, there was no difference in the functional connectivity between ACC and retrosplenial cortex and the depression-like behavior within the LPS group (Figure 6(c)). 
Right caudate putamen seed

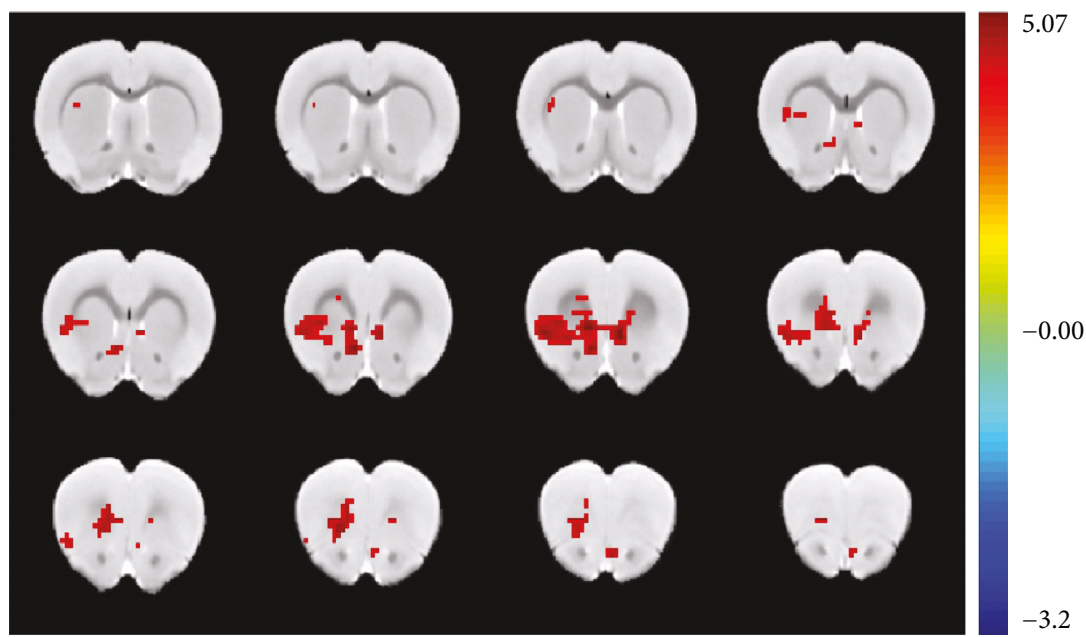

(a)

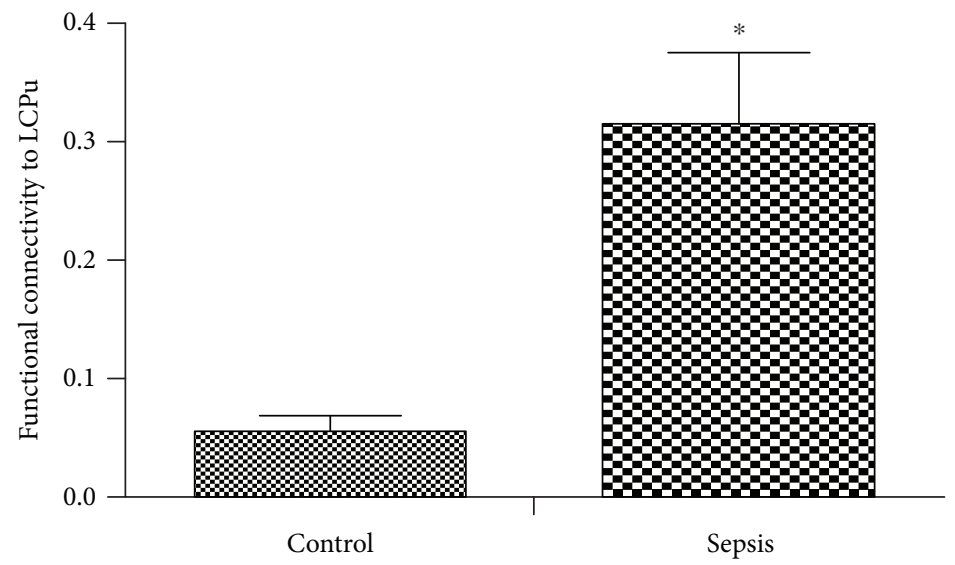

(b)

FIGURE 4: LPS challenge altered the functional connectivity between the right caudate putamen and left caudate putamen. (a) The group difference distribution map of the FC when the seed was set at the RCPu $(P<0.05$, corrected by family-wise error rate). Significantly increased FC are highlighted in the red-yellow heat bar scales. The RCPu seed showed increased functional connectivity with the left $\mathrm{CPu}$ compared with the control group. (b) The bar graph shows the mean FC $Z$ values of LCPu with the significant between-group difference extracted from the LPS and control groups. FC: functional connectivity; RCPu: right caudate putamen; LCPu: left caudate putamen. ${ }^{*} P<0.05$ vs. control group.

3.6. Correlation between Plasma Level of BDNF and Neuroimaging Data. Neither abnormal ReHo brain regions nor functional connectivity between relevant brain regions was associated with plasma level of BDNF (data not shown).

\section{Discussion}

In the present study, we found that LPS induced significantly increased ReHo values in the ACC and the CPu and increased functional connectivity within the reward, motivation, and emotion regulation network. The abnormal spontaneous neuronal activity in those areas provided information on the neural mechanisms underlying cognitive impairment and suggested that ReHo analysis might be a useful noninvasive imaging tool for the detection of early cerebral abnormalities in sepsis patients. To the best of our knowledge, this is the first study examining the brain functional connectivity changes in a lipopolysaccharide- (LPS-) induced systemic inflammation animal model using BOLD-based fMRI based on ReHo analysis.

Systemic inflammation induces physiological and behavioral changes in both humans and animals and is involved in mood and cognitive impairments [1-3]. During neuroinflammation, elevated brain cytokine levels can mediate sickness behaviors and alter cognitive processes [3-5]. By using an acute immune challenge, our study suggested that LPS induced significantly impaired mood impairment as reflected by depression-like behavior performed in the forced swim test, which is consistent with prior studies [16-18]. Our study along with previous findings supports the notion that experimental endotoxin administration constitutes an animal model to study the pathophysiology of SAE 


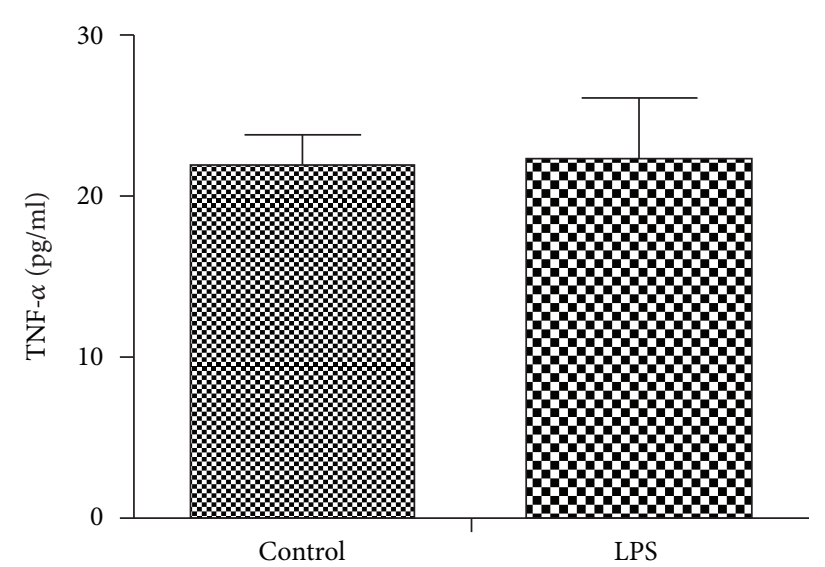

(a)

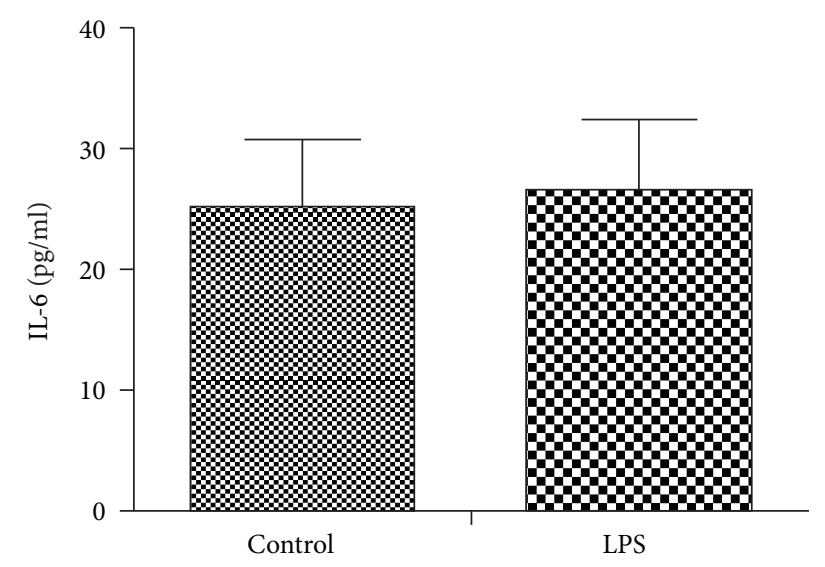

(c)

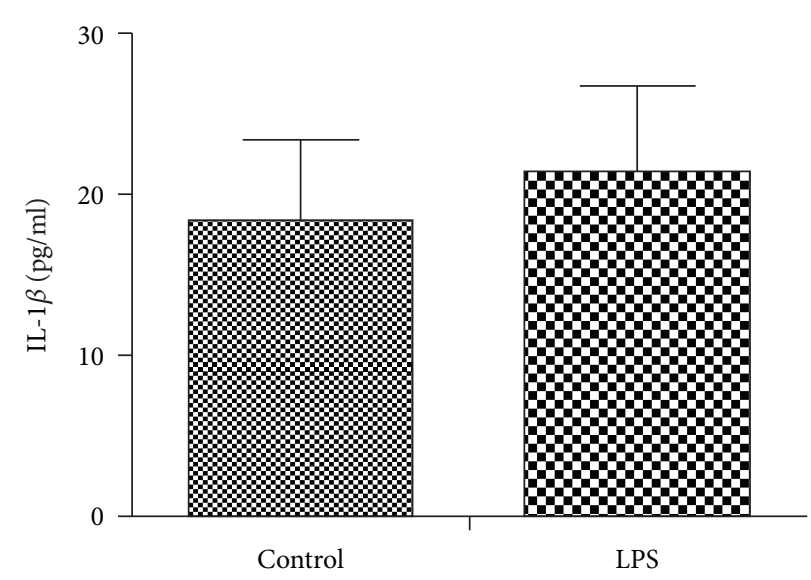

(b)

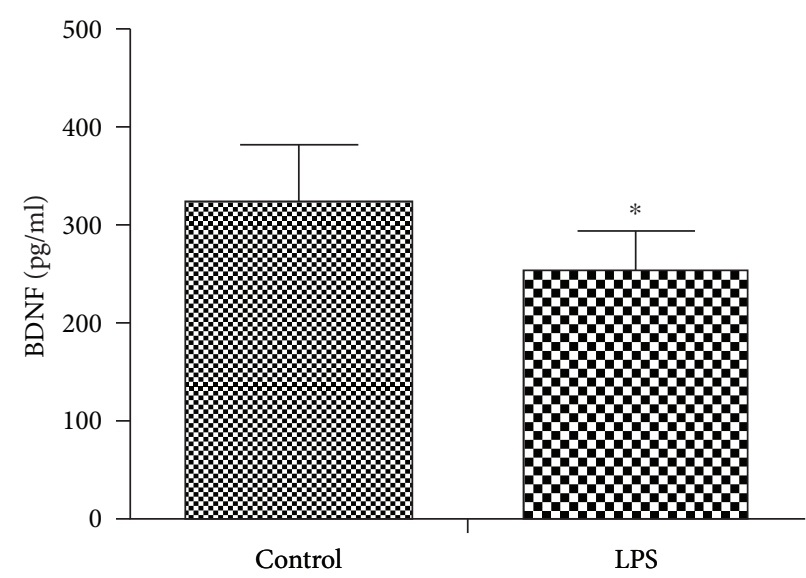

(d)

FIGURE 5: Effects of LPS challenge on plasma levels of proinflammatory mediators and BDNF. LPS challenge did not change the plasma proinflammatory mediators but decreased the BDNF levels. ${ }^{*} P<0.05$ vs. control group.

$[16,22,23]$. However, the neural mechanisms by which systemic inflammation induced mood impairment remain to be elucidated.

Functional neuroimaging is a useful technique for exploring the neurophysiological mechanisms underlying various clinical disorders [7-9]. It has been demonstrated that altered temporal connectivity of the BOLD signal between brain regions that form networks are important for cognition [7-9]. Thus, analyzing functional connectivity may reveal spontaneous fluctuations in brain networks during resting state and thus allow mapping the intrinsic functional organization between spatially remote brain areas [27]. However, it is difficult to recognize precisely which regions are altered and drive the large-scale alterations of network synchronization. In contrast to functional connectivity, ReHo reflects the changes in temporal aspects of spontaneous neuronal activity in a brain region. It can be used to identify aberrant coherence of local neural activity across the entire brain [18]. The ReHo method has been proved useful in detecting the regional changes in a variety of neurological diseases, including amnesic mild cognitive impairment [28], depression [29], and hepatic encephalopathy [30]. However, no imaging study has thus far been performed to address the brain functional connectivity changes after peripheral LPS challenge based on ReHo analysis. Using this approach, we found that LPS challenge induced a significant increase in ReHo values in the ACC and the CPu. The finding of increased ReHo in these regions might indicate that there might exist functional abnormality, a phenomenon that can be explained by the recruitment of compensatory mechanisms. Thus, our study supports the notion of the functional impairment during systemic inflammation from a new perspective of regional brain spontaneous activity.

Most of the symptomatic effects of sepsis such as affective and cognitive impairment can be attributed to inflammation $[2,3]$. However, our current study showed that LPS did not affect proinflammatory mediators when performed two days after LPS injection. One of the main reasons might be that the early cytokines such as TNF- $\alpha$, IL- $1 \beta$, and IL- 6 peak shortly after LPS injection and return to the baseline levels within hours [16], providing one explanation for the negative results observed in our study. Indeed, a number of studies have shown that proinflammatory cytokines and antiinflammatory cytokines return to normal after 24 hours after LPS stimulation $[16,31,32]$. Depression is one of the most common symptoms observed in sepsis patients $[2,3]$. Brain regions involved in this neural circuit such as the $\mathrm{CPu}$ (an anatomy that is similar to the human corpus 


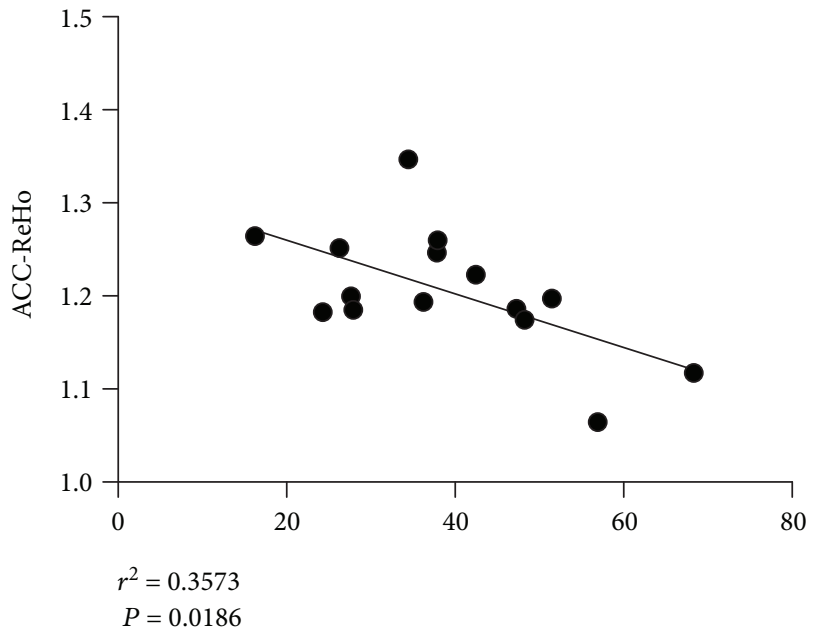

(a)

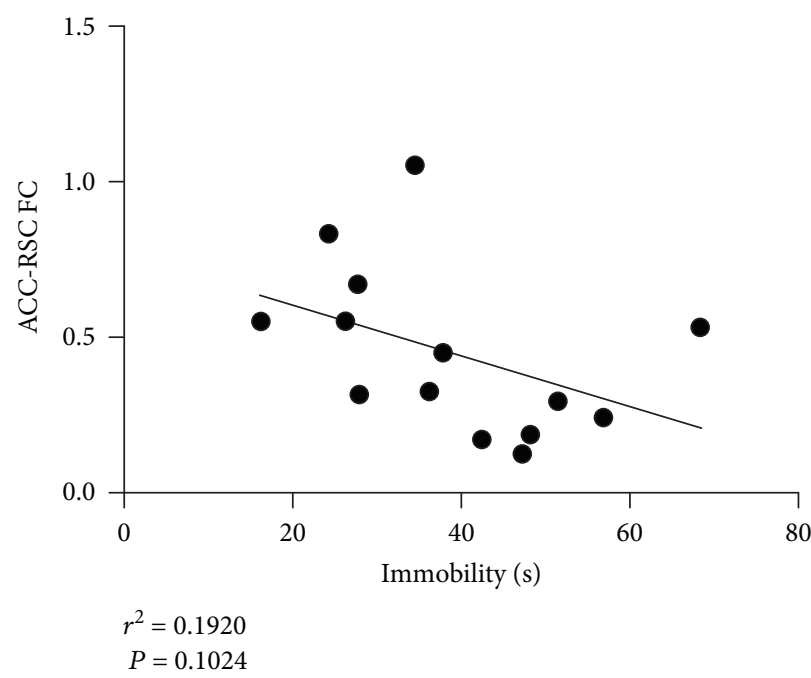

(c)

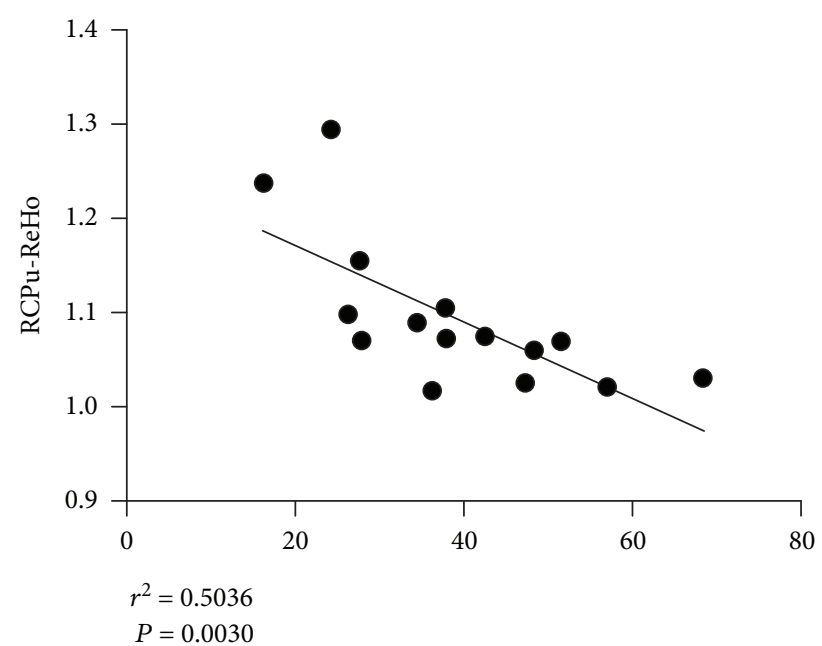

(b)

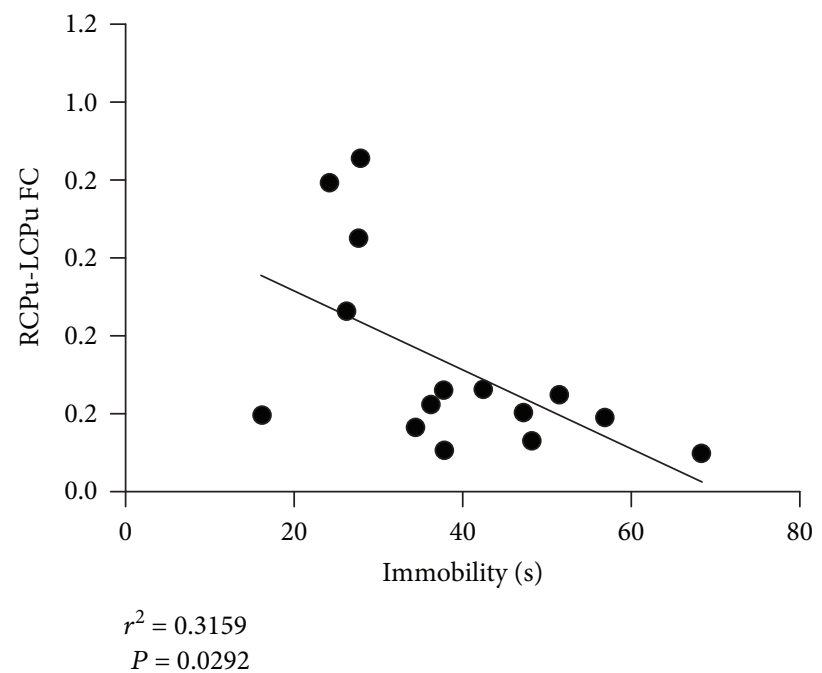

(d)

Figure 6: Correlation between depression-like behavior and neuroimaging data. The ReHo value in the two brain regions showed a correlation with depression-like behavior. The functional connectivity between the right $\mathrm{CPu}$ and the left $\mathrm{CPu}$ was significantly related to depression-like behavior. ACC: anterior cingulate cortex; LCPu: left caudate putamen; RCPu: right caudate putamen; RSC: retrosplenial cortex; FC: functional connectivity.

striatum), temporal lobe, amygdala, caudate, anterior cingulate cortex, and frontal cortex play a key role in the onset and/or maintenance of depression [33,34]. Accordingly, we showed that the ReHo values in the ACC and the $\mathrm{CPu}$ increased significantly after LPS challenge, suggestive of increased synchronization of regional neural activity in these areas. Our findings of enhanced LPS-induced activation of the ACC and $\mathrm{CPu}$ are of interest because these regions have been reported to play a vital role in regulation of affective, interoceptive, and reward processing [35-37]. Consistently, it has been suggested that the mood-regulating circuit is greater in depressed animals compared with controls [11]. In addition, previous neuroimaging research has demonstrated that the ACC and the $\mathrm{CPu}$ are relevant brain regions in potential neural networks that are affected by peripheral inflammatory challenge $[16,38,39]$. This was further supported by the negative relationship between ReHo values in the ACC and $\mathrm{CPu}$ and the depression-like behavior. Thus, the ReHo of the ACC and CPu may serve as a potential biological marker for SAE.

Cognitive deficits reflect not only the alteration of specific brain regions but also disturbances of functional connectivity between different brain regions. One of the ways to estimate the brain function at a global level is to measure functional connectivity between brain regions [7]. Previous neuroimaging studies have demonstrated that systemic inflammation alters the functional connectivity of many brain networks at rest $[16,17]$. Based on the ReHo analysis, we further suggested that LPS challenge induced significantly increased functional connectivity between the ACC and retrosplenial cortex. The involvement of the ACC and $\mathrm{CPu}$ in emotional processing and the interaction between emotion, reward, and cognition have been confirmed in a large number of studies [37-40]. Based on the previous cross-species 
cytoarchitectural mapping study, the rodent retrosplenial cortex can be related to the human precuneus [41], which is a key node of the default mode network (DMN). Given the functional regulation of the two higher-order brain structure, the ACC and retrosplenial cortex had been linked with negative emotional or behavioral symptoms. In addition, the abnormal alteration in functional connectivity between the ACC and retrosplenial cortex might potentially indicate disturbed DMN after LPS challenge. Thus, the impaired functionality of this region was correlated with depressionlike symptoms. In support, an altered connectivity within key DMN regions is observed in patients with major depression [40]. On the other hand, the striatal circuitry has distinct involvement in behavioral responses regarding rewards and losses [42]. In support, our study showed increased right $\mathrm{CPu}$ and left $\mathrm{CPu}$ connectivity after LPS challenge, which might explain the disruption in emotion regulation and motivational behaviors in depression. Notably, the correlation of functional connectivity between the right $\mathrm{CPu}$ and the left $\mathrm{CPu}$ and the depression-like behavior may further support the important role of the disrupted network in SAE. However, it should be noted that some discrepancies existed between our study and previous findings; a phenomenon might be attributed to differences in species, LPS dose, and time to perform the fMRI $[16,17]$. Together, our study that significantly increased ReHo value and functional connectivity in relevant brain regions suggested functional damage in SAE, but this speculation needs to be tested in future studies.

Some limitations should be mentioned in this study. Firstly, this is a cross-sectional study, which does not allow for causal conclusions about the relationship between alterations in functional connectivity and LPS-induced abnormal behaviors. Future longitudinal studies with antiinflammatory intervention are needed to clarify this association. Secondly, it is difficult to perform these experiments on conscious animals due to motion artifacts; the inevitable influence of anesthetization on neural function cannot be excluded. Because some experimental manipulations are unsafe or unethical to perform in humans, such as largerdose LPS challenge used in the present study, animal models are also necessary to improving our understanding of neurobiological processes that underlie systemic inflammationinduced mood and cognitive impairment. Finally, the relatively modest sample size may increase the risk of type II errors due to low statistical power.

To summarize, our study suggests that systemic inflammation induces mood impairment that is associated with abnormal regional spontaneous neuronal activity and resting-state functional network. Based on these data, this method may be used as an efficient way to assess inflammation effects on neurocircuitry in many psychiatric or medical illnesses associated with increased inflammation.

\section{Data Availability}

The data used to support the findings of this study are available from the corresponding author upon request.

\section{Conflicts of Interest}

The authors declare that they have no conflicts of interest.

\section{Acknowledgments}

This study was supported by the grants from the National Natural Science Foundation of China (Nos. 81771156 and 81772126).

\section{References}

[1] T. J. Iwashyna, E. W. Ely, D. M. Smith, and K. M. Langa, "Long-term cognitive impairment and functional disability among survivors of severe sepsis," Journal of the American Medical Association, vol. 304, no. 16, pp. 1787-1794, 2010.

[2] T. E. Gofton and G. B. Young, "Sepsis-associated encephalopathy," Nature Reviews Neurology, vol. 8, no. 10, pp. 557-566, 2012.

[3] S. T. Anderson, S. Commins, P. N. Moynagh, and A. N. Coogan, "Lipopolysaccharide-induced sepsis induces longlasting affective changes in the mouse," Brain, Behavior, and Immunity, vol. 43, pp. 98-109, 2015.

[4] M. H. Ji, L. L. Qiu, H. Tang et al., "Sepsis-induced selective parvalbumin interneuron phenotype loss and cognitive impairments may be mediated by NADPH oxidase 2 activation in mice," Journal of Neuroinflammation, vol. 12, no. 1, p. 182, 2015.

[5] J. Wu, L. Dong, M. Zhang et al., "Class I histone deacetylase inhibitor valproic acid reverses cognitive deficits in a mouse model of septic encephalopathy," Neurochemical Research, vol. 38, no. 11, pp. 2440-2449, 2013.

[6] J. Wu, M. Zhang, S. Hao et al., "Mitochondria-targeted peptide reverses mitochondrial dysfunction and cognitive deficits in sepsis-associated encephalopathy," Molecular Neurobiology, vol. 52, no. 1, pp. 783-791, 2015.

[7] X. Lei, Y. Wang, H. Yuan, and D. Mantini, "Neuronal oscillations and functional interactions between resting state networks," Human Brain Mapping, vol. 35, no. 7, pp. 35173528, 2014.

[8] R. M. Hutchison, T. Womelsdorf, J. S. Gati, L. S. Leung, R. S. Menon, and S. Everling, "Resting-state connectivity identifies distinct functional networks in macaque cingulate cortex," Cerebral Cortex, vol. 22, no. 6, pp. 1294-1308, 2012.

[9] J. N. Lee, E. W. Hsu, E. Rashkin et al., "Reliability of fMRI motor tasks in structures of the corticostriatal circuitry: implications for future studies and circuit function," Neuroimage, vol. 49, no. 2, pp. 1282-1288, 2010.

[10] D. Shah, J. Praet, A. Latif Hernandez et al., "Early pathologic amyloid induces hypersynchrony of BOLD resting-state networks in transgenic mice and provides an early therapeutic window before amyloid plaque deposition," Alzheimer's \& dementia, vol. 12, no. 9, pp. 964-976, 2016.

[11] J. Hui, G. Xi, S. Liu et al., "Blood oxygen level-dependent signals via fMRI in the mood-regulating circuit using two animal models of depression are reversed by chronic escitalopram treatment," Behavioural Brain Research, vol. 311, pp. $210-218,2016$.

[12] A. M. Belcher, C. C. Yen, H. Stepp et al., "Large-scale brain networks in the awake, truly resting marmoset monkey," Journal of neuroscience, vol. 33, no. 42, pp. 16796-16804, 2013. 
[13] M. Pievani, W. de Haan, T. Wu, W. W. Seeley, and G. B. Frisoni, "Functional network disruption in the degenerative dementias," The Lancet Neurology, vol. 10, no. 9, pp. 829843, 2011.

[14] G. S. Dichter, D. Gibbs, and M. J. Smoski, "A systematic review of relations between resting-state functional-MRI and treatment response in major depressive disorder," Journal of Affective Disorders, vol. 172, pp. 8-17, 2015.

[15] S. R. Kesler, M. Gugel, M. Pritchard-Berman et al., "Altered resting state functional connectivity in young survivors of acute lymphoblastic leukemia," Pediatric Blood \& Cancer, vol. 61, no. 7, pp. 1295-1299, 2014.

[16] F. Labrenz, K. Wrede, M. Forsting et al., "Alterations in functional connectivity of resting state networks during experimental endotoxemia - an exploratory study in healthy men," Brain, Behavior, and Immunity, vol. 54, pp. 17-26, 2016.

[17] M. Lekander, B. Karshikoff, E. Johansson et al., "Intrinsic functional connectivity of insular cortex and symptoms of sickness during acute experimental inflammation," Brain, Behavior, and Immunity, vol. 56, pp. 34-41, 2016.

[18] Y. Zang, T. Jiang, Y. Lu, Y. He, and L. Tian, "Regional homogeneity approach to fMRI data analysis," Neuroimage, vol. 22, no. 1, pp. 394-400, 2004.

[19] X. M. Kong, S. X. Xu, Y. Sun et al., "Electroconvulsive therapy changes the regional resting state function measured by regional homogeneity ( $\mathrm{ReHo}$ ) and amplitude of low frequency fluctuations (ALFF) in elderly major depressive disorder patients: an exploratory study," Psychiatry Research, vol. 264, pp. 13-21, 2017.

[20] P. Pan, H. Zhan, M. Xia, Y. Zhang, D. Guan, and Y. Xu, "Aberrant regional homogeneity in Parkinson's disease: a voxel-wise meta-analysis of resting-state functional magnetic resonance imaging studies," Neuroscience and Biobehavioral Reviews, vol. 72, pp. 223-231, 2017.

[21] C. Liu, Z. Xue, L. Palaniyappan et al., "Abnormally increased and incoherent resting-state activity is shared between patients with schizophrenia and their unaffected siblings," Schizophrenia Research, vol. 171, no. 1-3, pp. 158$165,2016$.

[22] N. Terrando, A. Rei Fidalgo, M. Vizcaychipi et al., "The impact of IL-1 modulation on the development of lipopolysaccharideinduced cognitive dysfunction," Critical Care, vol. 14, no. 3, article R88, 2010.

[23] A. R. Vasconcelos, L. M. Yshii, T. A. Viel et al., "Intermittent fasting attenuates lipopolysaccharide-induced neuroinflammation and memory impairment," Journal of Neuroinflammation, vol. 11, no. 1, p. 85, 2014.

[24] M. Ji, J. Xia, X. Tang, and J. Yang, "Altered functional connectivity within the default mode network in two animal models with opposing episodic memories," PLoS One, vol. 13, no. 9, article e0202661, 2018.

[25] M.-H. Ji, H. Tang, D. Luo et al., "Environmental conditions differentially affect neurobehavioral outcomes in a mouse model of sepsis-associated encephalopathy," Oncotarget, vol. 8, no. 47, pp. 82376-82389, 2017.

[26] M. H. Ji, Z. Y. Wang, X. R. Sun et al., "Repeated neonatal sevoflurane exposure-induced developmental delays of parvalbumin interneurons and cognitive impairments are reversed by environmental enrichment," Molecular Neurobiology, vol. 54, no. 5, pp. 3759-3770, 2017.
[27] K. C. Kern, S. M. Gold, B. Lee et al., "Thalamic-hippocampalprefrontal disruption in relapsing-remitting multiple sclerosis," Neuroimage Clinical, vol. 8, pp. 440-447, 2015.

[28] X. Luo, Y. Jiaerken, P. Huang et al., "Alteration of regional homogeneity and white matter hyperintensities in amnestic mild cognitive impairment subtypes are related to cognition and CSF biomarkers," Brain Imaging and Behavior, vol. 12, no. 1, pp. 188-200, 2018.

[29] Y. Liu, X. Zhao, Z. Cheng et al., "Regional homogeneity associated with overgeneral autobiographical memory of first-episode treatment-naive patients with major depressive disorder in the orbitofrontal cortex: a resting-state fMRI study," Journal of Affective Disorders, vol. 209, pp. 163-168, 2017.

[30] H. J. Chen, X. Q. Zhu, M. Yang et al., "Changes in the regional homogeneity of resting-state brain activity in minimal hepatic encephalopathy," Neuroscience Letters, vol. 507, no. 1, pp. 5-9, 2012.

[31] J. Lu, Q. Bao, D. Wang et al., "Effects of all-trans retinoic acid on lipopolysaccharide-induced synovial explant," Journal of Nutritional Science and Vitaminology, vol. 65, no. 1, pp. 8-18, 2018.

[32] T. Zhou, Y. Sun, Y. Wang et al., "Umbilical cord blood mesenchymal stem cells enhance lipopolysaccharide-induced IL-10 and IL-37 production in THP-1 cells," Inflammation, 2019.

[33] M. Ma, Q. Ren, C. Yang et al., "Antidepressant effects of combination of brexpiprazole and fluoxetine on depressionlike behavior and dendritic changes in mice after inflammation," Psychopharmacology, vol. 234, no. 4, pp. 525-533, 2017.

[34] J. J. Shaffer Jr, C. P. Johnson, J. G. Fiedorowicz, G. E. Christensen, J. A. Wemmie, and V. A. Magnotta, "Impaired sensory processing measured by functional MRI in Bipolar disorder manic and depressed mood states," Brain Imaging and Behavior, vol. 12, no. 3, pp. 837-847, 2018.

[35] J. Brakowski, S. Spinelli, N. Dörig et al., "Resting state brain network function in major depression - depression symptomatology, antidepressant treatment effects, future research," Journal of Psychiatric Research, vol. 92, pp. 147-159, 2017.

[36] R. J. Davidson, D. A. Lewis, L. B. Alloy et al., "Neural and behavioral substrates of mood and mood regulation," Biological Psychiatry, vol. 52, no. 6, pp. 478-502, 2002.

[37] J. C. Felger, Z. Li, E. Haroon et al., "Inflammation is associated with decreased functional connectivity within corticostriatal reward circuitry in depression," Molecular Psychiatry, vol. 21, no. 10, pp. 1358-1365, 2016.

[38] N. I. Eisenberger, E. T. Berkman, T. K. Inagaki, L. T. Rameson, N. M. Mashal, and M. R. Irwin, "Inflammation-induced anhedonia: endotoxin reduces ventral striatum responses to reward," Biological Psychiatry, vol. 68, no. 8, pp. 748-754, 2010.

[39] N. A. Harrison, L. Brydon, C. Walker, M. A. Gray, A. Steptoe, and H. D. Critchley, "Inflammation causes mood changes through alterations in subgenual cingulate activity and mesolimbic connectivity," Biological Psychiatry, vol. 66, no. 5, pp. 407-414, 2009.

[40] T. Wise, L. Marwood, A. M. Perkins et al., "Instability of default mode network connectivity in major depression: a two-sample confirmation study," Translational Psychiatry, vol. 7, no. 4, article e1105, 2017. 
[41] J. A. Ash, H. Lu, L. R. Taxier et al., "Functional connectivity with the retrosplenial cortex predicts cognitive aging in rats," Proceedings of the National Academy of Sciences of the United States of America, vol. 113, no. 43, pp. 12286-12291, 2016.

[42] C. Diener, C. Kuehner, W. Brusniak, B. Ubl, M. Wessa, and H. Flor, "A meta-analysis of neurofunctional imaging studies of emotion and cognition in major depression," Neuroimage, vol. 61, no. 3, pp. 677-685, 2018. 


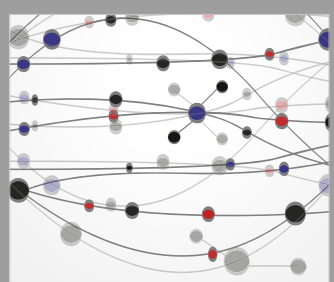

The Scientific World Journal
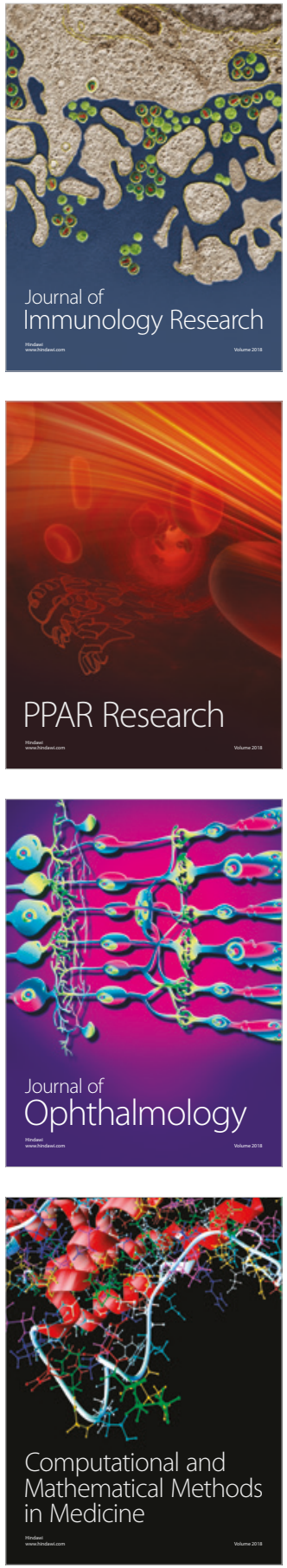

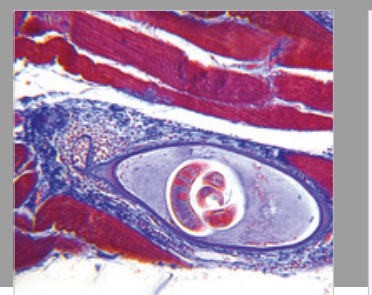

Gastroenterology Research and Practice

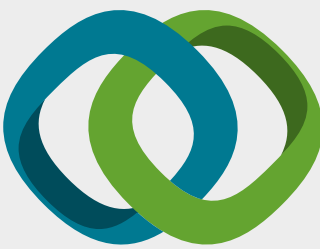

\section{Hindawi}

Submit your manuscripts at

www.hindawi.com
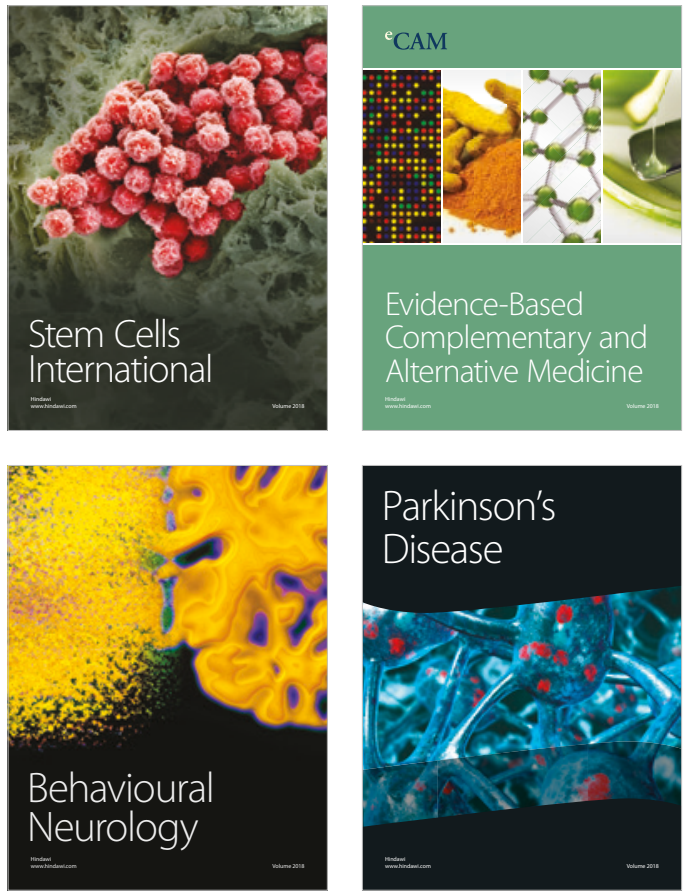

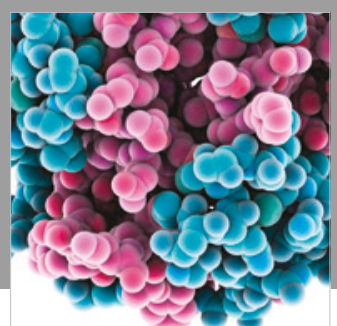

ournal of

Diabetes Research

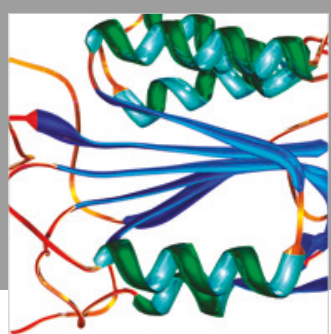

Disease Markers
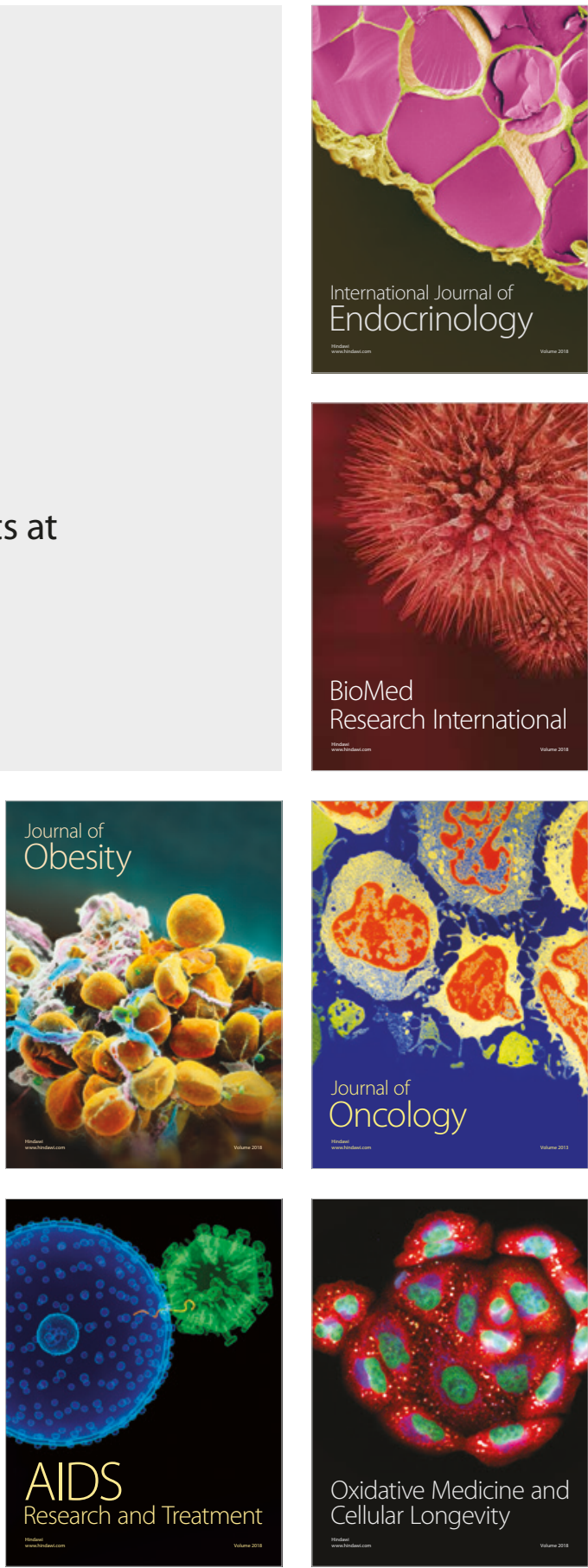